\title{
Minimum lesion detectability as a measure of PET system performance
}

Stephen Adler ${ }^{1 *}$ (D) Jurgen Seidel ${ }^{1}$, Peter Choyke², Michael V. Knopp ${ }^{3}$, Katherine Binzel ${ }^{3}$, Jun Zhang ${ }^{3}$, Craig Barker ${ }^{4}$, Shielah Conant ${ }^{4}$ and Roberto Maass-Moreno ${ }^{4}$

\begin{tabular}{l}
\hline * Correspondence: \\
stephen.adler@nih.gov \\
${ }^{1}$ Clinical Research Directorate/ \\
Clinical Monitoring Research \\
Program, Leidos Biomedical \\
Research, Inc., Frederick National \\
Laboratory for Cancer Research, \\
Frederick, Maryland 21702, USA \\
Full list of author information is \\
available at the end of the article
\end{tabular}

* Correspondence: stephen.adler@nih.gov Clinical Monitoring Research Program, Leidos Biomedical Research, Inc., Frederick National Laboratory for Cancer Research, Full list of author information is

\begin{abstract}
Background: A phantom in combination with an imaging protocol was developed to measure the limit of small lesion detection on different PET systems. Seven small spheres with inner diameters ranging from 3.95 up to $15.43 \mathrm{~mm}$ were imaged in a Jaszczak ECT Phantom, in air, in a cold background, and with sphere to background contrast ratios of 15:1 down to 1.88:1. The imaging times varied from 1 to $16 \mathrm{~min}$. The imaging protocol was performed on the Gemini TF and Vereos by Philips, the $\mathrm{mCT}$ and HRRT by Siemens, and the Discovery 710 by General Electric. For each scanning condition, the images were reconstructed with image voxel sizes of 1 to $4 \mathrm{~mm}$ cubic voxels. The reconstruction method used for each system was the one recommended by the manufacture to achieve best small image lesion detection results. A human observer study was performed to determine the smallest observable sphere for each scanning condition.
\end{abstract}

Results: All systems were able to image the smallest sphere of $3.95 \mathrm{~mm}$ inner diameter at the 15 to 1 signal to background ratio when imaged for 16 min. For a typical whole body per bed position scan time of 2 to 4 min, the smallest imaged sphere varied between 4.95 and $6.23 \mathrm{~mm}$ at the $15: 1$ contrast ratio and 12.43 and 15 . $43 \mathrm{~mm}$ at a contrast ratio of 1.88:1. In general, all systems were consistent with the Rose criteria when determining lesion detectability.

Conclusions: Besides demonstrating that the current state of the art clinical PET/CT systems have the same lesion detection ability, the study demonstrates how sensitive scan time can be to detecting small lesions which have a relatively small contrast uptake in the range of just 2:1. This should help guide imaging protocols to use longer scan times over regions of the subject in which small lesions are suspect.

Keywords: PET/CT imaging, Lesion detection, Human observer study, Signal to noise

\section{Background}

In the field of PET system development, there is a continuous effort in improving the performance of the next generation system. The technologies adopted and refined in PET systems address basic imaging parameters such as resolution, sensitivity, and aperture (the FOV both radially and axially) which affect the overall quality of the resulting PET image. Extensive research has gone into crystal design, time of flight technology, image reconstruction algorithms, and high speed data acquisition systems, to name a few areas of recent progress. A good reference is the book titled "Positron

(c) The Author(s). 2017 Open Access This article is distributed under the terms of the Creative Commons Attribution 4.0 International License (http://creativecommons.org/licenses/by/4.0/), which permits unrestricted use, distribution, and reproduction in any medium, provided you give appropriate credit to the original author(s) and the source, provide a link to the Creative Commons license, and indicate if changes were made. 
Emission Tomography" published by Springer [1] which covers all these aspects of the art and science of PET system design.

One of the great advantages of PET system technology is the ability to measure absolute radionuclide activity distributed throughout the field of view of the system. Therefore, besides improving image quality, efforts need to be directed towards image quantification or increasing the accuracy of radionuclide activity measurement. The goal is to measure with greatest accuracy the activity concentration of a radionuclidelabeled agent which has been injected into a human or non-human subject being scanned.

One side field of PET system or PET imaging assessment is that of lesion detection. The ability for a PET system to detect lesions is unique in that it brings to bear all the aspects of a PET system's features (sensitivity, resolution, reconstruction algorithms) to be able to detect small and/or dim lesions. A lot of effort has gone into the field studying the ability for a PET system to image lesions. These studies have focused on demonstrating improvements in either PET scan design or new image reconstruction techniques using improvements in lesion detection as the metric to assess success in the study at hand. Typically, these studies are performed on a single system and report the improvement attained in lesion detection by introducing a new imaging technology or improvements to reconstruction techniques. For example, how does the use of TOF improve lesion detection as compared to non-TOF. The same question is asked regarding the use of OSEM image reconstruction algorithm versus FBP. Surti, Schaefferkeotter, Larizen, Kadrams, El Fakhri, and Karp are some names of lead authors who have published in this field of study [2-12].

The breadth of research published on lesion detection in PET imaging highlights the importance of this topic and the interest within the molecular imaging oncology field. Being able to detect and measure lesions at their earliest stages of development plays a key role in the treatment staging of cancer patients.

The goal of this study was to assess a PET system's limit of lesion detection. This is in contrast as to whether a new technology is being validated by measuring the improvement of lesion detection which is the main focus of the publications cited above. The NEMA NU2 PET system performance tests stop short of performing a task which measures the limit of lesion detection. The NEMA NU2 test does provide a lesion imaging test in which hot spheres of different diameters are imaged in a warm background of a large volume phantom with a contrast ratio of either 8:1 or 4:1. All current clinical whole-body PET/CT systems are able to resolve the smallest sphere, thus leaving the main question to be how accurately the system can measure the activity concentration of the smallest sphere. This gives rise to using the contrast recovery coefficient as the metric to compare the imaging capability between systems.

This study attempts to take the NEMA NU2 image quality test to the next level by reducing the size of the spheres, increasing the number of sphere to background contrast settings, changing the scan times, and changing the reconstructed image voxel size to determine under what scanning conditions can a lesion of a certain size be positively observed. With this data set, one should be able to answer the following question. For a lesion of a given size, a tracer uptake to background of a given contrast reconstructed to a given image voxel size, will the lesion be positively observed? 
Furthermore, the signal to noise ratio (SNR) for each sphere under all scanning conditions and image reconstructions will be measured and correlated with the lesion observability of the human observer study. The purpose is to correlate the human observer study with the measured parameter SNR to validate the Rose criterion [13].

This article documents the method and results of this lesion detection limit study performed on the following systems; the Gemini TF [14] and Vereos [15] (Philips Medical Systems, Cleveland OH), the Discovery 710 [16] (General Electric Health Care, Waukesha WI) and the Biograph mCT [17], and HRRT [18] (Siemens Health Care, Knoxville TN).

\section{Methods}

\section{Phantom preparation}

We combined four small spheres from a set designed for pre-clinical scanners (ECT/ MI-HS/SET4, Data Spectrum Durham, NC) with the three smallest hollow spheres from a set made for clinical scanners (ECT/HS/SET6, Data Spectrum Durham, NC) for a total of seven spheres with inner diameters (and volumes) ranging from $3.95 \mathrm{~mm}$ $(31 \mu \mathrm{L})$ to $15.43 \mathrm{~mm}(2000 \mu \mathrm{L})$. The dimensions of these spheres were taken from the manufacturer's documentation and are listed in Table 1. These seven spheres were filled with an ${ }^{18} \mathrm{~F}$ solution and placed inside the Data Spectrum Jaszczak ECT Phantom and imaged under six different imaging conditions. First, the phantom was scanned in air, with no water in the phantom main chamber. Then in cold background, with the main phantom chamber filled with non-radioactive water and finally with four sphere to background contrast ratios of 15:1, 7.5:1, 3.75:1, and 1.88:1.

The ${ }^{18} \mathrm{~F}$ solution concentration used to fill the spheres and background was adjusted such that for the 3.75:1 contrast ratio scan; the background ${ }^{18} \mathrm{~F}$ solution concentration would be $143 \mathrm{nCi} / \mathrm{mL}$ or $5.29 \mathrm{kBq} / \mathrm{mL}$ at the start of the PET scan. This was done to match the background activity concentration specified by the NEMA NU2 image quality test. The background activity concentration was prepared using several syringes with a known amount of ${ }^{18} \mathrm{~F}$ activity. After each scan, the solution in each syringe was sequentially injected into the background volume of the phantom, thus changing the sphere to background ratio from the initial zero background activity to a ratio of 15 to $1,7.5$ to $1,3.75$ to 1 , and finally, 1.88 to 1 , with each new injection of activity from the prepared syringes. A detailed phantom preparation and imaging SOP is included in the Additional file 1 section.

Table 1 Table of sphere dimensions

\begin{tabular}{ll}
\hline Inner diameter $(\mathrm{mm})$ & Inner volume $(\mu \mathrm{L})$ \\
\hline 3.95 & 31 \\
4.95 & 63 \\
6.23 & 125 \\
7.87 & 250 \\
9.89 & 500 \\
12.43 & 1000 \\
15.43 & 2000 \\
\hline
\end{tabular}


For each of the six phantom preparations, the phantom was scanned for a total of $16 \mathrm{~min}$. From the $16 \mathrm{~min}$ of scanning data, images were reconstructed for the following scan times: $1,2,4,8$, and $16 \mathrm{~min}$. For each image reconstruction time, images were reconstructed to a target image voxel size of $1 \times 1 \times 1 \mathrm{~mm}(1 \mathrm{~mm}$ images), $2 \times 2 \times 2 \mathrm{~mm}$ ( $2 \mathrm{~mm}$ images), and $4 \times 4 \times 4 \mathrm{~mm}$ ( $4 \mathrm{~mm}$ images). Due to the voxel size constraint imposed by the reconstruction software for each system, when the target image voxel dimension was not possible, the closest one to the target dimension was selected. The actual image voxel size for each of the systems is shown in Table 2. One should note the large discrepancy in the target image voxel dimension of the Discovery 710 . Due to the manner in which the reconstruction software was written, the slice thickness cannot be changed from $3.27 \mathrm{~mm}$. Therefore, for the Discovery 710, all $Z$ dimensions are the same across all target voxel volumes. The PET images were generated using the manufacturer's recommended reconstruction settings. These settings are tabulated in Table 3.

In order to monitor the signal to background concentration ratio for each scan, the solution used to fill the spheres was sampled three times by pipetting $500 \mu \mathrm{L}$ of the solution into three separate vials. When the phantom was prepared for scanning at the various contrast ratios, the background volume of the phantom was sampled using a pipette to extract $500 \mu \mathrm{L}$ of the background volume and placed in vials. After the scanning was done, the radioactivity in the vials was counted in a Perkin Elmer gamma counter providing an independent measure of the signal to background contrast ratios.

\section{Human observer study}

In order to quantify lesion detectability, a human observer study was conducted. Two nuclear medicine physicians and one nuclear medicine technologist were recruited to view each image set and determined which of the 7 spheres they could observe. The observers were given a choice of three observation types to score the lesions with a value of 2, 1 , or 0 for lesions which were definitely observed, lesion observed but similar to other noise in the image, and not observed, respectively. Refer to Table 4 for a more detailed description of the lesion scoring scheme.

Each observer viewed the phantom images using the MIM display software system version 6.5 (MIM Software Inc, Cleveland OH, http://mimsoftware.com). The images were loaded on the viewing station in the three standard views of axial, sagittal, and coronal. The observer was allowed to change the contrast scale, zoom, and pan the images to best tune the images to score the spheres.

Table 2 Table of system image voxel dimensions. Note: $\mathrm{mCT} 1.07 \times 1.07 \times 1$ voxel volume is achieved through interpolation

\begin{tabular}{llll}
\hline \multicolumn{1}{c}{ Scanner } & \multicolumn{3}{c}{ Target Voxel Size $(\mathrm{mm})$} \\
\cline { 2 - 4 } & NA $1 \times 1 \times 1$ & $2 \times 2 \times 2$ & $4 \times 4 \times 4$ \\
\hline Gemini TF & $1 \times 1 \times 1$ & $2 \times 2 \times 2$ & $4 \times 4 \times 4$ \\
Vereos & $1 \times 1 \times 3.27$ & $2 \times 2 \times 3.27$ & $4 \times 4 \times 4$ \\
mCT & $1.07 \times 1.07 \times 1$ & $1.99 \times 1.99 \times 2$ & $4 \times 4 \times 3.27$ \\
HRRT & $1 \times 1 \times 1$ & $2 \times 2 \times 2$ & $4.07 \times 4.07 \times 4$ \\
\hline
\end{tabular}


Table 3 Reconstruction options used for each scanner

\begin{tabular}{ll}
\hline \multicolumn{1}{c}{ Scanner } & \multicolumn{1}{c}{ Reconstruction Parameters } \\
\hline Gemini TF & BLOB-OS-TOF, 33 subsets, 3 iterations, TOF kernel width $14.1 \mathrm{~cm}$, roll off 0.25 \\
Vereos & $\begin{array}{l}\text { 3D iterative, 29 subsets, } 3 \text { iterations, TOF, PSF regularization parameter 6.0, } \\
\text { Gaussian post-filter with 4.1-cm kernel. }\end{array}$ \\
Discovery 710 & $\begin{array}{l}\text { TOF-OSEM, 24 subsets, } 5 \text { iterations, PSF resolution } \\
\text { recovery, POst-filtered with 2-mm Gaussian in transaxial plane. }\end{array}$ \\
mCT & PSF + TOF OSEM, 21 subsets, 3 iterations, no postfiltering \\
HRRT & MOLAR [24], MAP-TR [25] \\
\hline
\end{tabular}

Each observer scanned all the image data sets which covered the five systems, four contrast to background settings, five scan times, and three image voxel sizes. All seven spheres for each image data set were scored and recorded for each observer in a database.

The observation scores recorded by the three human observers were aggregated to generate a detectability score of "sphere observed," "sphere not observed," or "sphere neither observed nor not observed." If all three observers scored the sphere with an observation score of 2 or two observers scored the sphere with an observation score of 2 and the third observer scored it with a value of 1 , then the sphere was scored as "observed." If all three observers gave the sphere an observation score of 0 or two observers gave the sphere a score of 0 and the third observer gave it a score of 1 , then the sphere was scored as "not observed." All other combinations of the three observation scores scored the sphere as "neither observed nor not observed."

\section{Signal to noise ratio measurement}

The signal to noise was measured for each sphere in all images generated in this study. The definition of the signal to noise used in this study is shown in the following equation: [19]

$$
\mathrm{SNR}=\frac{|\bar{s}-\bar{b}|}{\sigma_{b}}
$$

where $\bar{s}$ is the mean signal value, $\bar{b}$ is the mean background value, and $\sigma_{b}$ is the standard deviation of the background.

The images were processed using MIM image display system to measure the spatial coordinates of each of the spheres for all scans and in-house software algorithms written in $\mathrm{C}++$ were developed using root (CERN, Geneva Switzerland, http://root.cern.ch) to extract the SNR measurements for all spheres.

Table 4 Lesion detection scoring table

\begin{tabular}{ll}
\hline Score & Definition \\
\hline 2 & $\begin{array}{l}\text { If the sphere was a lesion, would the observation of the sphere affect the } \\
\text { course of treatment of the fictional subject } \\
1\end{array}$ \\
& $\begin{array}{l}\text { If the sphere was observable but could be similar to other noisy spots in } \\
\text { the image and would not qualify as a lesion which would affect the course } \\
\text { of the fictional patient's treatment. } \\
0\end{array}$ \\
\hline
\end{tabular}


The challenge with respect to the data analysis of this study is to know the locations of each of the seven spheres within each image. Because the scanning conditions were varied to such extremes, there are image data sets in which none of the spheres can be observed, and thus determining the location of the un-observable spheres within the background is difficult. To solve this problem, the scan in which the spheres were scanned in air was used to determine the relative location of the spheres within the Jaszczak phantom. Then, through a series of image registration steps, the locations of the spheres scanned in air were mapped to the spheres imaged in the four different contrast ratio scans. The details of the registration process can be found in the Additional file 1 .

To measure the signal for each sphere, a spherical contour was created which has the same inner diameter as the sphere at the center of the coordinates measured in the previous step. Within this contour, the maximum voxel value was recorded. Next, all the voxels which had a value of $80 \%$ of the maximum voxel value or greater were counted and recorded. Of this set of voxels which had values greater than $80 \%$ of the maximum, the mean was calculated and recorded.

To measure the warm background, an annulus region, $3 \mathrm{~cm}$ thick along the $z$-axis of the FOV, with a $3 \mathrm{~cm}$ inner diameter and $7 \mathrm{~cm}$ outer diameter in the transaxial plane, was defined and centered at the coordinates for each sphere. The mean and standard deviation of the background activity within this annulus region were measured and recorded.

The signal mean, background mean, and standard deviation were used to calculate the signal to noise ratio defined in Eq. 1.

\section{MIP image generation and visual contrast scaling procedure}

In order to display the images generated in this study for visual comparison between scanning conditions and across the systems, MIP images were generated with their gray scale set to normalize the noise across all images.

To generate the MIP images, the lowest and highest slices were determined along the patient bed $(Z)$ axis which contained all seven spheres. Next, a MIP images were formed using the lowest -2 slice through the highest +2 slice. The MIP image will then show the sphere data for all seven spheres in one two-dimensional slice. This procedure of finding the lowest and highest slices was necessary since the spheres do not all line up in a single plane. Also, the phantom was positioned manually on the patient bed and there was enough left or right tilt to move the smallest sphere outside the slice containing the center sphere. The phantom was positioned manually on the patient bed of the clinical PET/CT systems used in the study. Careful attention was paid to ensure the phantom was positioned rectilinearly aided by the laser positioning system of the system. But even so, there was a slight left/right tilt detected once the sphere location in the images was closely inspected.

Next, the mean and standard deviation on a pixel by pixel basis was measured in the area of the MIP image which has only the background data inside the phantom. The area of the MIP image where the spheres were located was excluded. The gray scale was then set such that white (gray scale 0 or minimum scale) was set at -10 standard deviations from the mean background and black (gray scale 256 or maximum scale) is set to +10 standard deviations above mean background. In this way, the visual gray scale of the images is normalized to the noise in the background. This scaling is 
important when visualizing the smallest lesion in imaging conditions in which the lesions are almost not detectable and allowing one to compare these hard to detect spheres across imaging conditions and systems.

\section{Results}

This study generated a total of 280 images based on images from five systems. This includes all variations of signal to background activity preparations, scan times, and voxel size reconstructions. Furthermore, adding in the seven spheres from each image, we have multiples of 1960 possible data points to present. To simplify the presentation of the data, only a representative sample is depicted here. But for completeness, all 280 images and all data values derived from the study are found in the Additional file 1. Figure 1 contains two bar graphs. The top graph shows the measured background activity measured from the PET image data at the start of the scan for each of the contrast preparations of 15:1, 7.5:1, 3.75:1, and 1.88:1. The bottom graph shows the contrast ratios achieved for each of the target contrast preparation using the pipetted samples extracted from the spheres and from the background volumes.

Table 5 tabulates the results of the human observer study determining the smallest visible sphere in microliters for all the scanning conditions for each of the five systems studied. Each column represents a different scan time in minutes going from 16, 8, 4, 2 , and $1 \mathrm{~min}$ from left to right. The entry NA means no images were available for that scanning condition and the entry NSV stands for no sphere visible. The results from
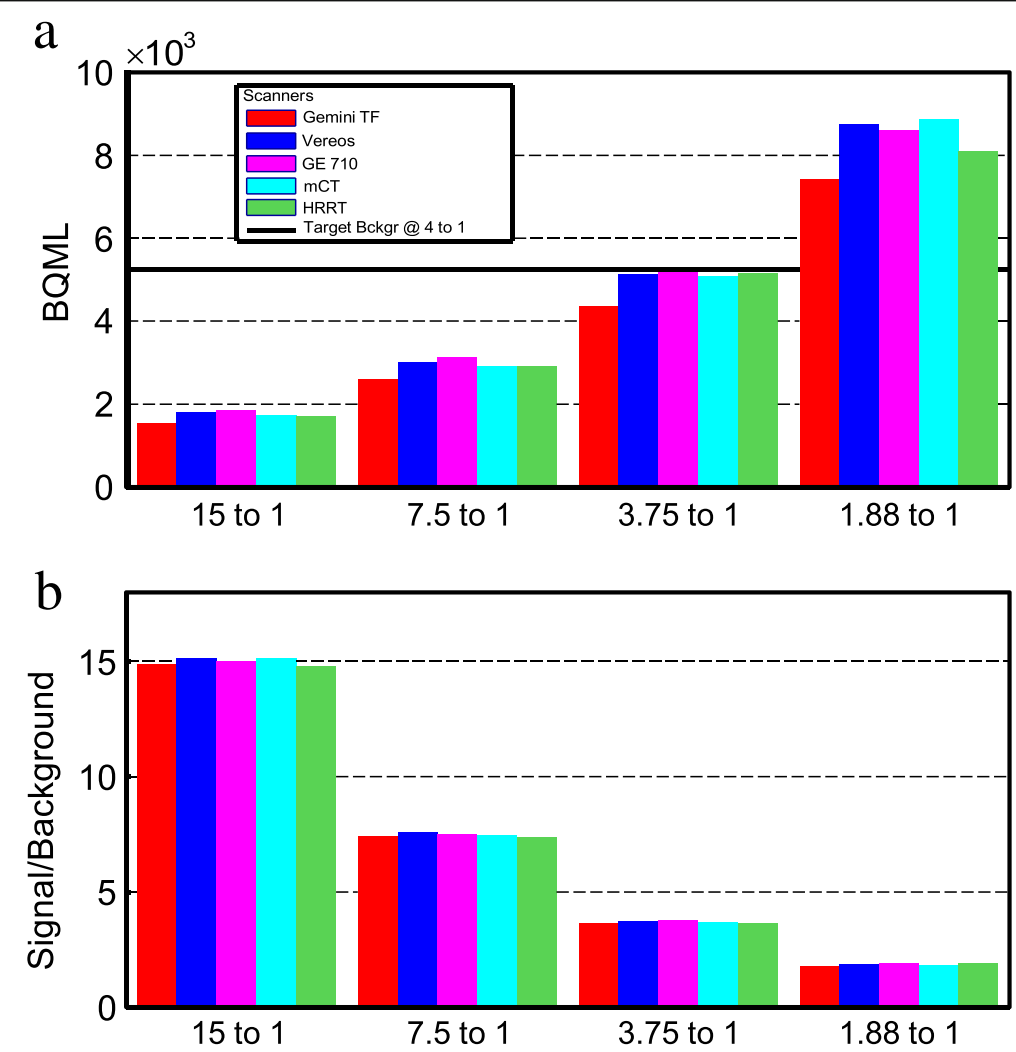

Fig. 1 Phantom preparation conditions for the warm background scans. The activity was measured using the PET imaging data and the sphere to background contrast was measured using the pipetted samples. a Measured background activity concentration. $\mathbf{b}$ Achieved sphere to background activity contrast 


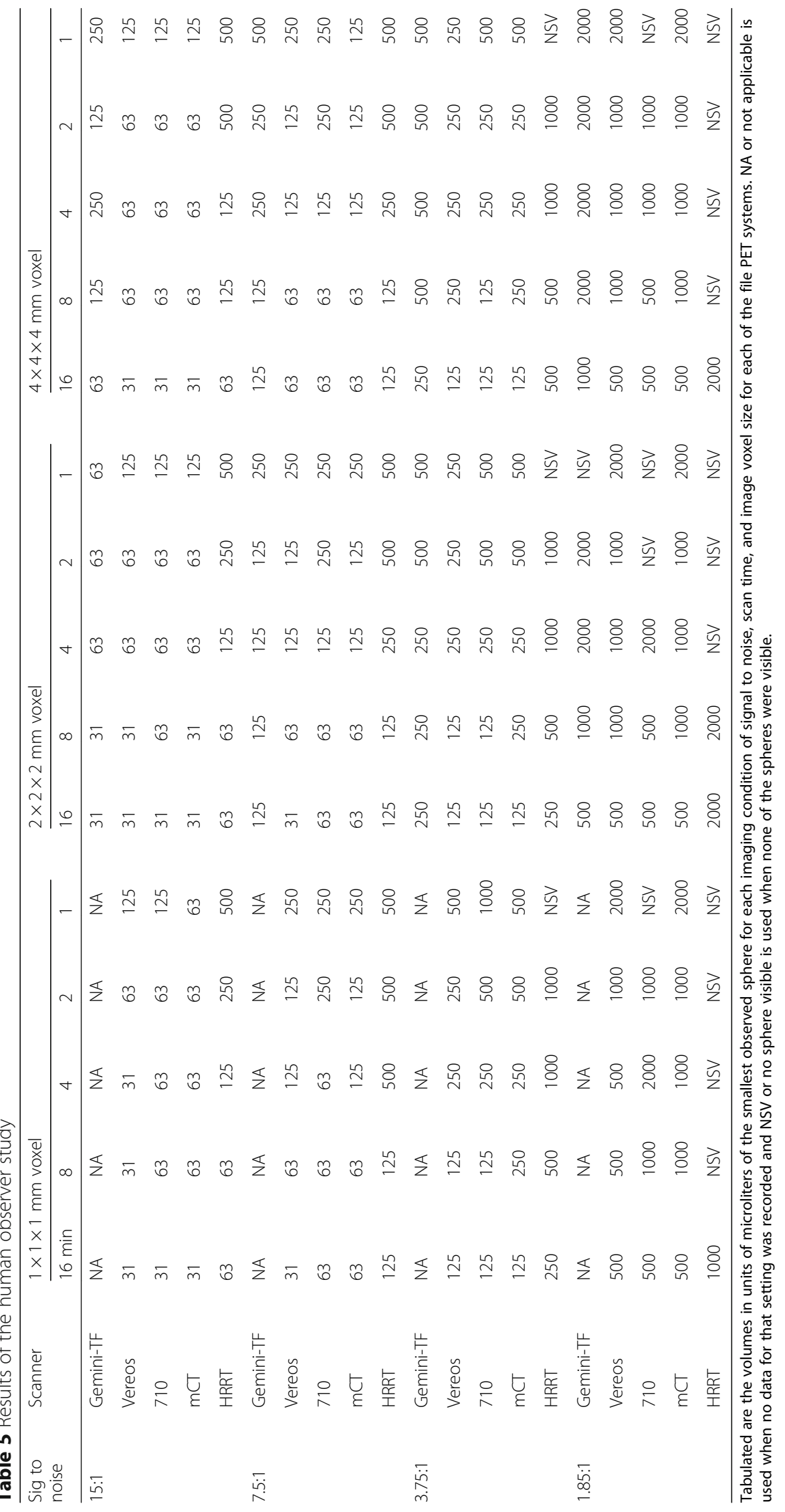


Table 6 Signal to noise ratio summary for 7.5:1 contrast sphere

\begin{tabular}{|c|c|c|c|c|c|c|c|c|c|c|c|c|c|c|c|c|}
\hline \multirow{2}{*}{$\begin{array}{l}\text { Scan } \\
\text { time }\end{array}$} & \multirow[t]{2}{*}{ Scanner } & \multicolumn{5}{|c|}{$\underline{1 \times 1 \times 1 \mathrm{~mm} \text { voxel }}$} & \multicolumn{5}{|c|}{$2 \times 2 \times 2 \mathrm{~mm}$ voxel } & \multicolumn{5}{|c|}{$\underline{4 \times 4 \times 4 \mathrm{~mm} \text { voxel }}$} \\
\hline & & $500 \mu \mathrm{L}$ & 250 & 125 & 63 & 31 & 500 & 250 & 125 & 63 & 31 & 500 & 250 & 125 & 63 & 31 \\
\hline \multirow[t]{5}{*}{$16 \mathrm{~min}$} & Gemini TF & NA & NA & NA & NA & NA & 15.1 & 12.4 & 9.8 & 5.4 & 5.6 & 78.5 & 32.9 & 18.5 & 5.6 & 0.6 \\
\hline & Vereos & 18.4 & 15.9 & 12.9 & 8.1 & 9.3 & 15.3 & 12.9 & 10.8 & 10.3 & 8.4 & 82.5 & 46.8 & 23.5 & 11.1 & 7.1 \\
\hline & 710 & 20.1 & 20.3 & 15.5 & 8.9 & 2.9 & 69.5 & 21.2 & 9.7 & 10.0 & 2.8 & 73.8 & 58.2 & 21.5 & 9.1 & 1.1 \\
\hline & $\mathrm{mCT}$ & 19.0 & 17.3 & 12.9 & 10.3 & 6.8 & 12.2 & 15.4 & 11.4 & 8.1 & 4.7 & 89.2 & 51.1 & 25.0 & 9.9 & 4.2 \\
\hline & HRRT & 18.0 & 15.9 & 12.6 & 10.9 & 4.4 & 10.5 & 13.8 & 10.2 & 7.7 & 1.9 & 32.5 & 20.1 & 11.1 & 3.9 & 0.0 \\
\hline \multirow[t]{5}{*}{$8 \mathrm{~min}$} & Gemi & NA & NA & NA & NA & NA & 15.7 & 12.0 & 8.9 & 4.4 & 2.3 & 28.7 & 14.2 & 10.3 & 2.3 & 1.7 \\
\hline & Verec & 18.0 & 15.4 & 12.3 & 6.9 & 7.1 & 15.8 & 13.0 & 10.1 & 6.7 & 5.8 & 57.7 & 26.4 & 14.6 & 8.6 & 4.7 \\
\hline & 710 & 16.9 & 18.6 & 13.9 & 7.7 & 1.4 & 73.2 & 17.7 & 18.4 & 6.9 & 1.0 & 47.3 & 42.4 & 12.2 & 6.1 & 0.2 \\
\hline & $\mathrm{mCT}$ & 19.0 & 16.3 & 13.4 & 9.5 & 3.6 & 12.7 & 15.1 & 11.5 & 7.2 & 3.4 & 67.7 & 40.8 & 20.4 & 8.8 & 2.4 \\
\hline & HRRT & 177 & 15.1 & 10.1 & 7 & 3.6 & 14.9 & 15.2 & 10.0 & 5.3 & 1.6 & 21.5 & 1 & 9.2 & 2.6 & 1.3 \\
\hline \multirow[t]{5}{*}{$4 \mathrm{~min}$} & Gemi & NA & NA & NA & NA & NA & 13.8 & 10.3 & 7.7 & 3.5 & 2.5 & 22.9 & 9.6 & 5.3 & 2.9 & 1.3 \\
\hline & Vereo & 17.4 & 14.3 & 10.6 & 5.9 & 6.0 & 14.4 & 12.9 & 9.3 & 4.0 & 4.9 & 38.2 & 22.1 & 10.3 & 5.6 & 2.4 \\
\hline & 710 & 17.2 & 14.6 & 14.0 & 8.4 & 4.8 & 11.5 & 12.0 & 30.7 & 8.5 & 4.5 & 39.7 & 27.1 & 12.2 & 8.4 & 1.7 \\
\hline & $\mathrm{mCT}$ & 18.4 & 16.6 & 10.1 & 6.5 & 3.7 & 16.6 & 12.9 & 9.5 & 5.1 & 2.9 & 55.6 & 28.7 & 10.8 & 4.3 & 1.7 \\
\hline & HRRT & 16 & 14.9 & 1 & 55 & 2. & 12.5 & 31.1 & 20.5 & 1 & 1.5 & 14.3 & 85 & 68 & 3.6 & 0.8 \\
\hline \multirow[t]{5}{*}{$2 \min$} & Gemini TF & NA & NA & NA & NA & NA & 11.7 & 13.8 & 8.4 & 3.4 & 1.5 & 14.8 & 8.2 & 4.3 & 3.2 & 0.5 \\
\hline & Vereo & 16.2 & 13.6 & 7.0 & 3.8 & 5.5 & 14.8 & 12.7 & 6.6 & 2.2 & 4.2 & 28.6 & 13.9 & 10.2 & 3.8 & 1.1. \\
\hline & 710 & 14.4 & 14.9 & 8.8 & 4.9 & 1.5 & 58.8 & 17.2 & 6.8 & 4.5 & 0.0 & 27.9 & 19.1 & 6.3 & 0.9 & 0.0 \\
\hline & $\mathrm{mCT}$ & 18.4 & 16.5 & 10.8 & 5.1 & 6.9 & 19.6 & 13.0 & 11.1 & 3.7 & 6.9 & 32.4 & 17.7 & 10.4 & 2.6 & 51 \\
\hline & HRRT & 15.3 & 14.5 & 11.0 & 3.3 & 2.1 & 12.8 & 13.4 & 8.5 & 2.4 & 0.2 & 12.7 & g & 5.6 & 0.5 & 0 \\
\hline \multirow[t]{5}{*}{$1 \mathrm{~min}$} & Gemini TF & NA & NA & NA & NA & NA & 13.0 & 11.0 & 4.7 & 3.0 & 0.9 & 10.2 & 4.3 & 2.3 & 0.2 & 0.5 \\
\hline & Vereos & 14.5 & 11.8 & 8.6 & 3.8 & 4.2 & 13.4 & 12.0 & 7.8 & 1.9 & 2.9 & 22.9 & 11.3 & 6.3 & 4.0 & 1.1 \\
\hline & 710 & 14.8 & 13.3 & 10.7 & 2.0 & 2.8 & 14.0 & 13.5 & 7.2 & 1.6 & 1.1 & 18.8 & 20.2 & 10.9 & 1.8 & 0.5 \\
\hline & $\mathrm{mCT}$ & 17.2 & 11.4 & 9.3 & 5.3 & 1.2 & 12.4 & 10.2 & 7.4 & 4.3 & 0.5 & 25.8 & 11.9 & 8.3 & 2.7 & 0.3 \\
\hline & HRRT & 13.9 & 14.9 & 7.7 & 3.9 & 9.7 & 9.4 & 12.1 & 7.3 & 1.4 & 3.2 & 11.1 & 3.9 & 1.6 & 0.5 & 2.2 \\
\hline
\end{tabular}

the human observer study show that all systems were able to image the smallest microsphere of $3.95 \mathrm{~mm}$ inner diameter at the 15 to 1 signal to background ratio when imaged for $16 \mathrm{~min}$. For a typical whole body per bed position scan time of 2 to $4 \mathrm{~min}$, the smallest imaged sphere varied between 4.95 and $6.23 \mathrm{~mm}$ at the $15: 1$ contrast ratio and 12.43 and $15.43 \mathrm{~mm}$ at a contrast ratio of 1.88:1.

Table 6 tabulates the SNR measured for a subset of the scans. The purpose of the table is to give one a comparison of SNR values across the five systems which participated in this study. The data for the 7.5:1 contrast ratio scan and the five smallest spheres are presented therein. A full compilation of the SNR values can be found in the Additional file 1.

With the tabulated human observer data and the SNR values for each sphere, one can correlate minimum lesion detectability as determined by a human observer with the SNR measured for each sphere. To examine this correlation, a histogram of SNR for minimally observed spheres and for non-observed spheres is present, one for each system in Fig. 2. The two sets of spheres, the observable ones and the non-observable ones, are shown to be separated from each other with the observable spheres in general having a SNR greater than 5 and the non-observable spheres having a SNR less than 5 . 


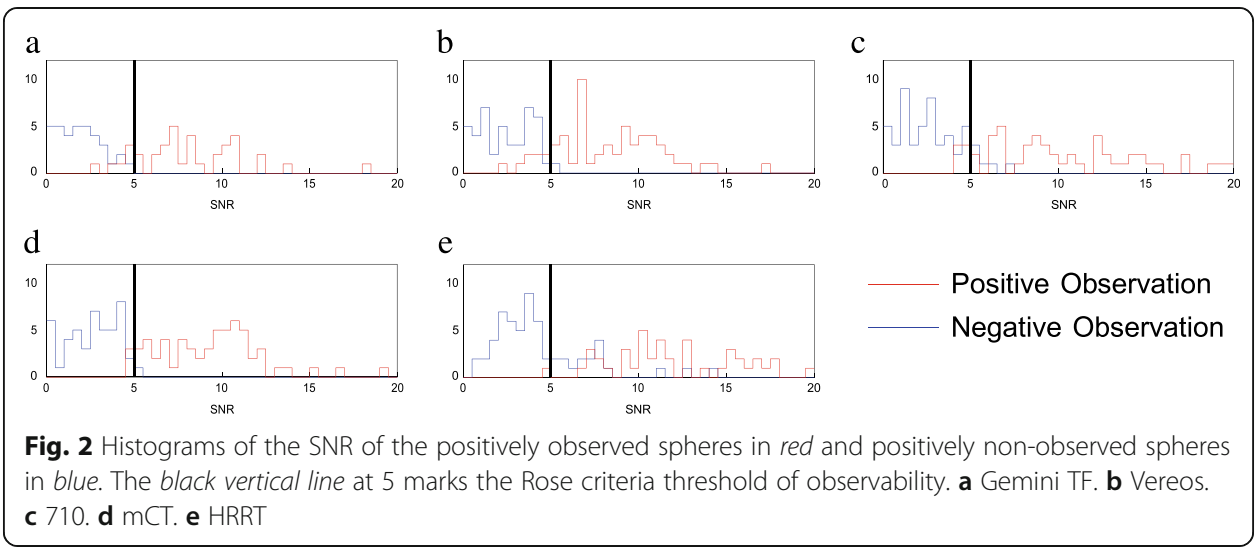

This agrees with the Rose criterion named after Albert Rose, who studied object observability back in the 1970s. He established an observability criterion such that for a small object to be observed, it had to have a SNR greater than 5 [13].

The final data presentation summary is a select set of images of the spheres which can be found in Figs. 3 and 4. Figure 3 gives a side by side visual comparison of the 7.5:1 signal to background preparation for the five systems. All images represent a 16-min acquisition and reconstructed to the three target voxel sizes of $1 \times 1 \times 1 \mathrm{~mm}, 2 \times 2 \times 2 \mathrm{~mm}$, and $4 \times 4 \times$ $4 \mathrm{~mm}$. The images in Fig. 4 show images of the phantom for a 15:1 signal to background phantom preparation. The top row is 2 -min scans and the bottom row is 16 -min scans.

\section{Discussion}

The goal of the study was to survey the imaging parameter space of lesion to background contrast ratios, scan time, image voxel size, and lesion volume in order to determine a system's limit of detecting small lesions. The idea is to measure this important clinical capability which is not addressed by conventional phantoms. Typical "resolution" phantoms rely on high contrast objects whereas the "real world" task is to detect relatively lower contrast objects. Keep in mind the NEMA NU2 image quality test, the smallest hot sphere has an inner diameter of $10 \mathrm{~mm}$. This sphere size is too large to measure the limits of detection.

For the comparison of minimum lesion detectability across systems, it was vital to ensure that the phantom preparation was consistent so that each system imaged the phantom at the same signal to background concentration ratio and absolute activity in the field of view at the start of each scan. The data presented in Fig. 1 establish that the

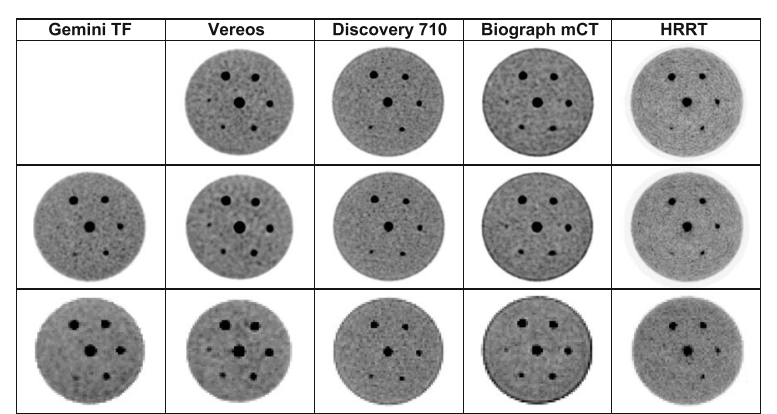

Fig. 3 Scanner comparison, 7.5 to 1 contrast ratio. Top row contains 1-mm voxel reconstructions, middle row is $2 \mathrm{~mm}$ voxel reconstructions and last row is $4-\mathrm{mm}$ voxel reconstructions. All images are 16-min scans 


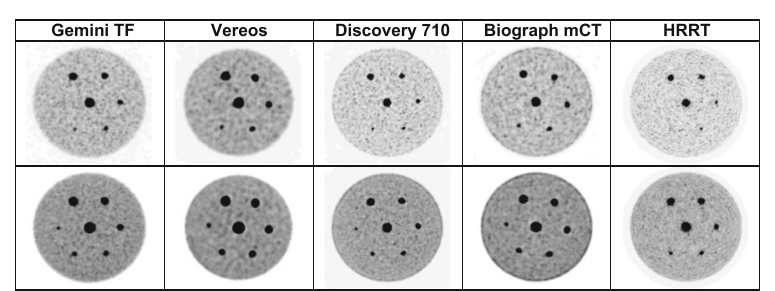

Fig. 4 Scanner comparison, 15 to 1 contrast ratio. Top row is 2-min acquisitions, bottom row is 16-min acquisitions. All images were reconstructed to $2 \mathrm{~mm}$ voxel size

phantom was prepared consistently for each system. Note that the absolute activity for the Philips Gemini TF is lower by $14 \%$ on average between each of the 4 concentration ratios. This small deviation of lower absolute activity should have a minimal affect by slightly reducing the ability to image the smallest lesion. The difference is considered to be negligible since the concentration ratios were all within $2 \%$ of each other as seen in the bottom plot of Fig. 1.

With the phantom data acquired on the five systems, the next step was to reconstruct the images in a manner which would be best suited to compare the system's ability to image small lesions. Since PET reconstruction algorithms are complex and implemented in a unique way on each system, it was left to each system operator to use whatever setting and algorithms were available to generate what the manufacturer would consider the best reconstruction settings to generate the best quality images. Therefore, the number of iterations, subsets, and postfiltering parameters which can play a role in adjusting the image quality of the system was not specified. See Table 3 which tabulates the reconstructions settings used for each system. The only specification for image generation was the voxel size of $1 \times 1 \times 1 \mathrm{~mm}, 2 \times 2 \times 2 \mathrm{~mm}$, and $4 \times 4 \times 4 \mathrm{~mm}$.

This brings us to the main result of the study, which is to determine the minimum lesion size which is observable under the different scanning conditions explored by this study. The main results are found in Table 5. This table lists which was the smallest detected sphere from the human observer study for each scan and image reconstruction condition and elucidates some expected results. The longer one scans, the smaller the lesion that can be observed. The higher the signal to background contrast ratio, the smaller the lesion can be observed. A more subtle effect is the image voxel size. One can pick out some cases in which for the same scanning condition of scan time and contrast ratio, changing the voxel size, will allow one to observe a smaller lesion. For example, the Vereos system demonstrates that for a 15:1 signal to background contrast ratio and a scan time of $8 \mathrm{~min}$; it can detect the $31 \mu \mathrm{L}$ sphere with a $2 \times 2 \times 2 \mathrm{~mm}$ voxel image which cannot be done on a $4 \times 4 \times 4 \mathrm{~mm}$ voxel image. Searching through Table 5 , one can find other examples of improved lesion detection when an image is reconstructed using a smaller voxel size. This effect of improving the lesion detectability of the system by reconstructing the images with a smaller voxel size has also been published by Morey et al. [20]. Kadrmas et al. [21] have published data showing improving lesion detection as one increases scan time.

The change in lesion detection for different voxel size reconstructions is also a case study in partial volume effects. Soret et al. [22] have a very nice discussion on how pixel or voxel size partial volume effects for small lesions affect the reconstructed lesion. 
Mainly for small lesions under two to three full-width at half maximum resolution of the scanner, the larger the full-width at half maximum resolution of the system, the impact on lesion detection is greater the larger the pixel or voxel size is, with the larger voxels degrading the lesion due to spill-out effects. Due to the warm background, there are larger spill-in effects as well. The data set acquired for this study will provide for a good analysis of partial volume effects, especially since the study focuses on small lesions which push the resolution limits of the systems. The results from a full study on partial volume effects will be published in a separate manuscript.

If we were to summarily state an overall result for this study, this would be that for a typical 2 to 4 min scan time per bed position, one can detect a lesion as small as $0.5 \mathrm{~cm}$ in diameter if the contrast to background ratio is $15: 1$ or better. For lesions with a contrast to background ratio of at least 2:1, the smallest lesion that one can detect will be $1.5 \mathrm{~cm}$ in diameter.

Table 6 and the accompanying Table 5 list the SNR values for a subset of images and give a more fine-grained measure of how well the system was able to resolve a lesion. But one should be careful not to rely on a few units of SNR to claim one system can better resolve a lesion scanned under a given condition than another.

In a not very surprising confirmation of the Rose criterion, the histograms shown in Fig. 2 show how observable lesions and non-observable lesions fall on either side of the dark line drawn at a SNR of 5.

The final set of data presented are the actual images of the phantoms so one can gauge via visual inspection the detectability of the spheres for a few selected imaging conditions. Figure 3 displays the maximum intensity projection (MIP) images for the slices which contain any of the seven spheres. The goal is to limit the number of slices over which one applies the MIP algorithm to only those which have sphere data and thus reduce the overall noise in the warm background volume of the phantom. Refer to the "Methods" section where a more detailed explanation of how the MIP images were prepared for display in Figs. 3 and 4. The images are for each system for the 7.5 to 1 signal to background contrast ratio. The top row is for $1 \times 1 \times 1 \mathrm{~mm}$ voxel images, the second row for $2 \times 2 \times$ $2 \mathrm{~mm}$ voxel images, and the last row for $4 \times 4 \times 4 \mathrm{~mm}$ voxel images. In this case, one can see how the voxel size can affect lesion detectability due to voxel partial volume effects as discussed earlier. Notice in the HRRT data, how the $63 \mu \mathrm{L}$ sphere at the 7 o'clock position is clearly visible in the top row $(1 \times 1 \times 1 \mathrm{~mm}$ voxel image) and is not present in the bottom row $(4 \times 4 \times 4 \mathrm{~mm}$ voxel image).

Figure 4 shows images for different scan times. These are the 15 to 1 signal to background concentration ratio images. The top row is the 1-min scan time images and the bottom row is the 16-min scan time images. All images were reconstructed to $2 \mathrm{~mm}$ voxel size. In most cases, the smallest lesion at the 9 o'clock location cannot be resolved in the 1min acquisition time images while it can be resolved in the 16-min acquisition time images. The exception is the HRRT scanner in which the smallest lesion was not detected at all.

To complete the discussion, the shortcomings of the study design should be mentioned. The first one is that the spheres are all in the same location removing the element of random placement thus giving the human observer a known location to search for the lesion. The other disadvantage is that the spheres have relatively thick plastic shells which will decrease the actual observability. This has been shown by 
Berthon et al. [23] and should be taken into account. Another drawback of the study is that the diameter of the Jazczak QC PET phantom used in this study is not comparable to a human torso. Studies have shown that lesion detectability varies greatly as a function of subject BMI as noted by Karp et al. [6]. Therefore, one should not take the results listed in Table 5 as absolute but perhaps as a lower limit.

For the reader who wishes to do a full examination of all the image data generated by this study, all the images can be found in the Additional file 1 section.

\title{
Conclusions
}

The publication of the data presented herein is the result of a comprehensive study of PET/CT systems undertaken by the NCI Molecular Imaging Program. The goal was to assess the current state of the art of PET/CT systems. Typically, one would perform a literature search and tabulate the NEMA NU2 measurements for each PET/CT system. Here, we took one step further and performed scans in all systems available using a single phantom that would best challenge the PET/CT system design. The study of how small a lesion a PET/CT system could image was chosen as the main outcome since this parameter is one that is clinically relevant in cancer detection.

\section{Additional file}

Additional file 1: Minimum lesion detectability as a measure of PET scanner performance. (PDF 21569 kb)

\begin{abstract}
Acknowledgements
The authors would like to thank the participation of Xiao Jin and Chuck Sterns from GE for their help in preparing and scanning the phantom used for this study on the GE Discovery 710, along with all the image reconstructions which are presented herein. The authors would also like to thank Dr Liza Lindenberg and Philip Eclarinal of the NCI/MIP and Dr. Ethan S. Bergvall, Defense Health Agency, Fort Belvoir Community Hospital, for taking part in the human observer study.
\end{abstract}

\section{Funding}

This project has been funded in whole or in part with federal funds from the National Cancer Institute, National Institutes of Health, under Contract No. HHSN261200800001E. The content of this publication does not necessarily reflect the views or policies of the Department of Health and Human Services, nor does mention of trade names, commercial products, or organizations imply endorsement by the U.S. Government.

\begin{abstract}
Authors' contributions
SA headed the study, oversaw the phantom data acquisition on all scanners, designed the human observer study, wrote the analysis software, generated all plots and figures, and wrote the main draft of the manuscript and supplemental materials section. JS helped design the phantom study. PC instigated and authorized funding for the study. MK provided facilities and resources for the data collected on the Verios system. KB participated in the data collection and image reconstruction work for the Verios system. JZ participated in the data collection and image reconstruction work for the Verios system. CB and SC participated in the data collection and image reconstruction work for the HRRT system. RM participated in the data collection and image reconstruction work for the mCT system. All authors read and approved the final manuscript.
\end{abstract}

Competing interests

The authors declare that they have no competing interests.

Ethics approval and consent to participate

This article does not contain any studies with human participants or animals performed by any of the authors.

\section{Author details}

${ }^{1}$ Clinical Research Directorate/Clinical Monitoring Research Program, Leidos Biomedical Research, Inc., Frederick National Laboratory for Cancer Research, Frederick, Maryland 21702, USA. ${ }^{2}$ Molecular Imaging Program, Center for Cancer Research, National Cancer Institute, Bethesda, USA. ${ }^{3}$ Department of Radiology, The Ohio State University Wexner Medical Center, Columbus, OH 43210, USA. ${ }^{4}$ Positron Emission Tomography Department, Warren G. Magnuson Clinical Center, National Institutes of Health, Bethesda, USA. 
Received: 15 October 2016 Accepted: 8 February 2017

\section{Published online: 04 March 2017}

\section{References}

1. Bailey DL, Townsend DW, Valk PE, Maisey MN. Positron emission tomography. Berlin: Springer; 2005.

2. Surti S, Scheuermann J, El Fakhri G, Daube-Witherspoon ME, Lim R, Abi-Hatem N, Mous-sallem E, Benard F, Mankoff D, Karp JS. Impact of time-of-flight pet on whole-body oncologic studies: a human observer lesion detection and localization study. J Nucl Med. 2011;52(5):712719.

3. Lartizien C, Kinahan PE, Swensson R, Comtat C, Lin M, Villemagne V, Trbossen R. Evaluating image reconstruction methods for tumor detection in 3-dimensional whole-body pet oncology imaging. J Nucl Med. 2003:44(2):276290

4. Lartizien C, Kinahan PE, Comtat C. A lesion detection observer study comparing 2-dimensional versus fully 3-dimensional whole-body pet imaging protocols. J Nucl Med. 2004;45(4):714-23.

5. Kadrmas DJ, Casey ME, Conti M, Jakoby BW, Lois C, Townsend DW. Impact of time-of-flight on PET tumor detection. J Nucl Med. 2009;50(8):13151323.

6. Karp JS, Surti S, Daube-Witherspoon ME, Muehllehner G. Benefit of time-of-flight in pet: experimental and clinical results. J Nucl Med. 2008;49(3):462470.

7. Lois C, Jakoby BW, Long MJ, Hubner KF, Barker DW, Casey ME, Conti M, Panin VY, Kadrmas DJ, Townsend DW. An assessment of the impact of incorporating time-of-flight information into clinical PET/CT imaging. J Nucl Med. 2010;51(2):237245

8. El Fakhri G, Surti S, Trott CM, Scheuermann J, Karp JS. Improvement in lesion detection with whole-body oncologic time-of-flight pet. J Nucl Med. 2011;52(3):347353.

9. Metz CE. Roc analysis in medical imaging: a tutorial review of the literature. Radiol Phys Technol. 2007;1 (1):212. doi:10.1007/s12194-007-0002-1.

10. Schaefferkoetter J, Casey M, Townsend D, El Fakhri G. Clinical impact of time-of-flight and point response modeling in pet reconstructions: a lesion detection study. Phys Med Biol. 2013;58(5):1465.

11. El Fakhri G, Santos PA, Badawi RD, Holdsworth CH, Van Den Abbeele AD, Kijewski MF. Impact of acquisition geometry, image processing, and patient size on lesion detection in whole-body 18F-FDG pet. J Nucl Med. 2007; 48(12):19511960.

12. Gifford H, Kinahan P, Lartizien C, King M. Evaluation of multiclass model observers in PET LROC studies. IEEE Trans Nucl Sci. 2007;54(1):116123.

13. Rose A. Vision: human and electronic. Optical physics and engineering. New York: Plenum Press; 1973.

14. Surti S, Kuhn A, Werner ME, Perkins AE, Kolthammer J, Karp JS. Performance of Philips Gemini TF PET/CT scanner with special consideration for its time-of-flight imaging capabilities. J Nucl Med. 2007;48(3):471480.

15. Nguyen NC, Vercher-Conejero JL, Sattar A, Miller MA, Maniawski PJ, Jordan DW, Muzic RF, Su KH, ODonnell JK, Faulhaber PF. Image quality and diagnostic performance of a digital PET prototype in patients with oncologic diseases: initial experience and comparison with analog PET. J Nucl Med. 2015;56(9):13781385.

16. Bettinardi V, Presotto L, Rapisarda E, Picchio M, Gianolli L, Gilardi MC. Physical performance of the new hybrid PETCT Discovery-690. Med Phys. 2011;38(10):53945411.

17. Jakoby BW, Bercier Y, Conti M, Casey ME, Bendriem B, Townsend DW. Physical and clinical performance of the mCT time-of-flight PET/CT scanner. Phys Med Biol. 2011;56(8):23752389.

18. Wienhard K, Schmand M, Casey ME, Baker K, Bao J, Eriksson L, Jones WF, Knoess C, Lenox M, Lercher M, Luk P, Michel C, Reed JH, Richerzhagen N, Treffert J, Vollmar S, Young JW, Heiss WD, Nutt R. The ECAT HRRT: performance and first clinical application of the new high resolution research tomograph. IEEE Trans Nucl Sci. 2002:49(1):104110. doi:10.1109/TNS.2002.998689.

19. Bushberg JT. The essential physics of medical imaging. Lippincott Williams \& Wilkins; 2002.

20. Morey A, Noo F, Kadrmas D. Effect of using $2 \mathrm{~mm}$ voxels on observer performance for pet lesion detection. J NUCL MED MEETING ABSTRACTS 55(1 MeetingAbstracts), 2093. 2014

21. Kadrmas DJ, Oktay MB, Casey ME, Hamill JJ. Effect of scan time on oncologic lesion detection in whole-body PET. IEEE Trans Nucl Sci. 2012;59(5):19401947.

22. Soret M, Bacharach SL, Buvat I. Partial-volume effect in PET tumor imaging. J Nucl Med. 2007:48(6):932945

23. Berthon B, Marshall C, Edwards A, Evans M, Spezi E. Influence of cold walls on pet image quantification and volume segmentation: a phantom study. Med Phys. 2013;40(8):082505.

24. Carson RE, Barker WC, Liow J-S, Johnson CA. Design of a motion-compensation OSEM list-mode algorithm for resolution-recovery reconstruction for the HRRT. In: Nuclear Science Symposium Confer- ence Record vol. 5. IEEE; 2003. pp. 32813285.

25. Sibomana M, Byars L, Panin V, Lenox M, Kehren F, Rist J, Burbar Z, Michel C, Knoess C, de Jong HWAM. Simultaneous measurement of transmission and emission contamination using a collimated 137Cs point source for the HRRT. In: Nuclear Science Symposium Conference Record vol. 4. IEEE; 2004. pp. 264726514. doi:10.1109/NSSMIC.2004.1462795 